\title{
INVESTIGACIONES
}

\section{Problemas de convivencia escolar desde la mirada del alumnado de educación secundaria}

\author{
School life problems from the perspective of secondary education students
}

\author{
Martha Leticia Gaeta González ${ }^{a}$, Valentín Martínez-Otero Pérez, \\ Maximiliano Rodrigo Vega ${ }^{b}$, Manuel Rodrigo Gómez ${ }^{c}$ \\ ${ }^{a}$ Facultad de Educación, UPAEP Universidad, México. \\ marthaleticia.gaeta@upaep.mx \\ ${ }^{b}$ Facultad de Educación, Universidad Complutense de Madrid, España. \\ valenmop@edu.ucm.es, mrodrig1@edu.ucm.es \\ ${ }^{c}$ Dirección General de Seguridad Ciudadana, Policía Nacional, España. \\ mrodrigo74kz@policia.es
}

\section{RESUMEN}

En este trabajo se analizan los principales problemas de convivencia escolar relacionados con los menores de edad, desde la visión del alumnado de Educación Secundaria Obligatoria (ESO). Participaron 116 alumnos de $3^{\circ}$ de ESO, de cuatro Institutos españoles, que respondieron un instrumento diseñado ad hoc. Los resultados muestran que el acoso, el ciberacoso y la violencia de género están presentes en los centros escolares y, en lo que refiere al racismo, más de la mitad de los alumnos han sido testigos de agresiones de este tipo. Tres de cada cuatro alumnos han presenciado actos violentos en los entornos escolares. El consumo de alcohol y en menor grado el de drogas tienen presencia en los centros. Esperamos que la exploración de los datos contribuya a dar un tratamiento educativo para la mejora de la convivencia escolar.

Palabras claves: victimización, racismo, violencia, violencia escolar, vicio.

\section{ABSTRACT}

This paper analyzes the school coexistence main problems of related to minors from Compulsory Secondary Education (ESO) students' vision. Participants were 116 students from 3rd grade of ESO, belonging to four Spanish Institutes, who responded to an instrument designed ad hoc. Results show that bullying, cyberbullying, and gender-based violence are present in schools, and, regarding racism, more than half of students have witnessed this type of aggression. Three out of four students have witnessed violent acts in school settings. The consumption of alcohol and, to a lesser extent, that of drugs are present in the centers. We hope that the exploration of these data will contribute to an educational treatment to improve school life.

Key words: victimization, racism, violence, school violence, addiction. 


\section{INTRODUCCIÓN}

Hablar de convivencia es tanto como referirse a la vida en compañía de otros. Valga esta pequeña definición para advertir que la vida humana sólo es posible merced a la participación de los demás. Como bien nos recuerda Marías (1996, p. 39), la vida personal es necesariamente interpersonal, es decir, convivencia. Sin embargo, esta naturaleza convivencial del hombre no impide lamentablemente que las relaciones sociales puedan debilitarse, romperse o perturbarse de forma considerable. Esto es precisamente lo que sucede cuando hablamos de conflictos en las relaciones humanas, ya sean de orden familiar, laboral, escolar o de cualquier otra modalidad.

Aun cuando la institución escolar debiera ser ámbito de convivencia, no de mera coexistencia, lo cierto es que con frecuencia se rompe la paz en los centros educativos hasta el punto de hacer imposible la formación. Con esta atmósfera de conflicto, de violencia manifiesta, la labor educativa se torna quimera. Como bien afirman Bravo y Herrera (2011), los centros escolares no son contextos fáciles para la convivencia, acaso por la progresiva complejidad que está adquiriendo la institución escolar en su conjunto, reflejo de la sociedad en que se inserta.

En este trabajo nos ocupamos de indagar en los posibles conflictos que acontecen en los centros escolares, sobre todo de los que se producen entre el alumnado en plena adolescencia en Educación Secundaria Obligatoria, ya sea por situaciones de acoso o ciberacoso, por fenómenos tales como el racismo, el vandalismo o la violencia de género, o por consumo de alcohol u otras drogas. Conscientes de la dificultad de abordar en toda su extensión el fenómeno de la conflictividad escolar en sus diversificadas manifestaciones nos acercamos de forma exploratoria, desde una perspectiva socio-contextual, a los problemas de convivencia en los centros educativos con el propósito de conocer mejor esta realidad a partir de la percepción del propio alumnado y, al mismo tiempo, ver qué posibles caminos nos conducen a sus soluciones.

\subsection{COMPORTAMIENTOS ANTISOCIALES EN LOS CENTROS ESCOLARES}

La violencia es la fuerza (proviene del latín vis = fuerza) que se ejerce en contra de otra $\mathrm{u}$ otras personas. Estas acciones intencionales orientadas a dañar al prójimo admiten gradación. En el ámbito escolar, por ejemplo, encontrarnos agresiones físicas a compañeros y educadores que varían en intensidad; robos, deterioro intencionado de material, insultos, burlas, amenazas, etc. Éstas serían algunas manifestaciones de la violencia física o psicológica que más se repiten en los centros escolares. Hay casos en los que la situación se vuelve insostenible y algunos profesores y alumnos temen ir al centro.

Estas conductas perturban el proceso educativo de todos hasta el punto de que la seguridad escolar se ha convertido en objeto de preocupación internacional. Desde la UNESCO-IICBA (2017), por ejemplo, se afirma que las escuelas seguras, con climas positivos, contribuyen al desarrollo físico, social, emocional y cognitivo de los estudiantes, así como a la salud y el bienestar de los docentes y del personal.

¿A qué se debe la violencia escolar? Este problema obedece a un conjunto de factores (pluricausalidad). Por un lado, hay que tener presente que la escuela ha experimentado grandes cambios con el aumento de la escolaridad de la enseñanza. Es frecuente oír, por ejemplo, que la positiva extensión de la escolaridad obligatoria comporta un incremento 
de los alumnos desmotivados e indisciplinados. Por otro, los medios de comunicación, y en particular la televisión y el mundo cibernético, ejercen sobre los escolares una gran influencia, a menudo perniciosa.

Tampoco hay que olvidar que estamos en una sociedad con altas dosis de violencia y que la escuela no es un campo aislado. Si hay violencia en la familia, en la calle y en los medios, lo extraño sería que no se manifestase también en los centros escolares. Esta aseveración no es una justificación, sino una constatación de la interdependencia de los diversos ámbitos -como bien han descrito los modelos sistémico y ecológico-, así como de la complejidad de la violencia que, stricto sensu, exigiría un análisis macroscópico -al menos inicial y teórico-, aunque por razones prácticas nos centremos principalmente en el sistema escolar.

La mayoría de los autores están de acuerdo en que la violencia escolar es atribuible a un conjunto de posibles causas, y la interacción de todas ellas posibilita su interpretación y posibles modelos de intervención y prevención (Gázquez et al., 2015; Penalva, 2018). La violencia, en general, es el resultado de una enmarañada red de factores entre los que sobresalen los sociales/ambientales, los mediáticos, los familiares, los escolares, los relacionales y los personales. En la medida en que se den cita factores correspondientes a los distintos niveles mencionados es más fácil que el menor muestre conductas antisociales. Por otro lado, la experiencia alcanzada en materia de prevención y corrección de la violencia escolar permite adelantar algunas consideraciones de carácter general que se sintetizan en el enriquecimiento de la comunicación interpersonal y la integración de los alumnos en las actividades de la comunidad educativa. Hay que avanzar en la construcción de centros escolares humanizados erigidos sobre la racionalidad y la afectividad, en los que ha de cumplir un papel fundamental la participación de las familias (Martínez-Otero, 2018; Sánchez, Daura y Laudadío, 2019).

Algunos de los comportamientos que más preocupan en nuestros días son los relativos al acoso y al ciberacoso. El acoso escolar o bullying puede definirse como una persecución física o psicológica intencionada y continua, que recibe un alumno por parte de otro u otros. Molina y Vecina (2015) destacan algunas características del bullying: la indefensión de la víctima; la ausencia de provocación; el desequilibrio de poder entre agresor y víctima; la complicidad, pasividad o ignorancia del entorno, al igual que la persistencia. Un fenómeno en el que, como indica Castillo-Pulido (2011), intervienen numerosos factores asociados con los orígenes, contexto sociocultural, entorno familiar y nivel socioeconómico de los sujetos involucrados y de la institución escolar que los alberga. Entre las modalidades de bullying que, como dice Nicolás (2011), suelen presentarse de modo simultáneo, encontramos agresiones físicas, humillaciones, amenazas, desprecio y exclusión.

El impacto del acoso puede ser grave y duradero tanto en los escolares que lo sufren como en los que lo infligen y en los que lo observan. Aunque el fenómeno del acoso escolar no es nuevo, sí es mucho más reciente su manifestación en internet, el llamado "ciberacoso" (del inglés cyberbullying). Se trata de una emergente forma de hostigamiento que a través de medios electrónicos, principalmente internet y el teléfono móvil (Garaigordobil, 2011), realiza un grupo o un sujeto contra una víctima con escasas posibilidades de defenderse. Por supuesto, este tipo de acoso, intencional y repetido, también puede coexistir con el acoso tradicional, lo que supone que algunos alumnos después de sufrirlo en el colegio siguen siendo perseguidos (amenazados, insultados, vejados...) a través de las tecnologías, a veces de forma anónima, cuando están en su casa o en cualquier otro lugar. 
En una investigación sobre el ciberacoso realizada en Portugal por Caetano et al. (2017) a partir de 3525 adolescentes escolarizados, se constató que las causas más invocadas por los agresores para explicar su conducta son, por un lado, de índole hedonista, relacionadas con juegos, diversión y con la evitación del aburrimiento, y, por otro lado, las que tienen que ver con su pertenencia a un determinado grupo o con inadecuadas conductas reactivas. En cuanto a los que se identifican como víctimas, atribuyen los comportamientos de sus agresores a su pertenencia a ciertos grupos, así como a la búsqueda de placer y de poder $\mathrm{y}$, en concreto, a aspectos como la envidia, la diversión, la inmadurez, los celos, la falta de respeto, la ausencia de afecto y los sentimientos de superioridad.

Cerezo-Ramírez (2012) destaca la sensación que tiene la víctima de no poder escapar de las agresiones, pues en cualquier lugar puede recibir mensajes amenazantes y, además, a la vista de muchos. Aunque no tienen por qué coexistir, no es nada extraño que la victimización mediante acoso presencial acontezca también a través de dispositivos electrónicos. El acoso y el ciberacoso, que generan gran preocupación escolar y social, afectan de modo creciente a ambos géneros y a centros escolares de titularidad pública y privada. A priori, ningún colegio o instituto se libra de su zarpazo y puede asegurarse con carácter general que cuando en la institución, del tipo que fuere, hay un compromiso auténtico con la convivencia, que se extiende a la familia, y que se patentiza en el cultivo de valores favorecedores de la relación interpersonal, estos problemas disminuyen.

En lo que se refiere al vandalismo escolar, se puede definir a partir de Torner (2016), como un conjunto de conductas, a menudo realizadas en grupo, mediante las que se destruyen o deterioran bienes materiales de las instituciones: cristales, cerraduras, incendios, pintadas, mesas y sillas, equipos informáticos, etc. Vilalta y Fondevila (2018), por su parte, han encontrado asociación entre el vandalismo escolar con la pertenencia a pandillas, el uso de drogas, la intimidación, la falta de apego a las normas institucionales escolares y el desigual trato dispensado por los profesores a sus alumnos.

Entre los comportamientos antisociales en los centros escolares, además de los mencionados, y que también serán objeto de esta investigación, se hallan los que producen discriminación u otras formas de maltrato a personas o grupos por motivos raciales o por la nacionalidad. La sociedad, en general, y la escuela, en particular, son cada vez más multiculturales, lo que hace que en mayor grado pueda saltar la chispa de la intolerancia en forma de racismo o xenofobia. Estos comportamientos ocupan hoy por derecho propio un lugar entre las conductas antisociales en la escuela. Por desgracia, están a la orden del día las noticias que dan cuenta de actos de violencia que tienen como protagonistas a escolares y a familiares intolerantes con algunos alumnos, ya sea por el color de la piel, el país de origen, la cultura, etc. Recientemente, Ibarra (2018) en el editorial del Informe Raxen consignaba que nos hallamos ante la "mundialización de la intolerancia" y que se extiende el rechazo al mestizaje y a la interculturalidad, que crece el racismo y la xenofobia, que se fomenta la etno-diferencia y que aparecen múltiples expresiones identitarias que se viven desde conductas de fanatismo. Fenómenos aberrantes que, en cualquier caso, han de prevenirse también desde la escuela porque, como afirma García-Medina (2018), los espacios de convivencia escolar deben asumir el principio de inclusión educativa, la educación intercultural y la lucha expresa y decidida contra cualquier forma de intolerancia.

Igualmente condenable y llamada a prevenirse es la violencia de género, sobre la que no siempre hay datos porque lamentablemente en ocasiones pasa inadvertida. Es un tipo de violencia «invisible» que sufre más la población femenina que la masculina y que, por 
lo común, se concreta en hostigamientos, tocamientos y, en menor medida, en violaciones. Ruiz-Ramírez y Ayala-Carrillo (2016) sostienen que las agresiones por violencia de género no se presentan de forma única y aislada, sino que se pueden exhibir diferentes agresiones a la vez, lo cual perjudica gravemente la salud, autoestima y auto-percepción de la víctima y también de sus familias.

Por su parte, Arenas (2013) a partir de su investigación concluye que hay una socialización asociada a un sesgo sexista que genera desiguales conductas y creencias, lo que da lugar, en su manifestación más grave, a malos tratos entre las parejas de adolescentes. Por esto, se requiere también en este aspecto el concurso de toda la comunidad educativa y el compromiso de fomentar la educación para todos, verdaderamente inclusiva. Aun cuando la violencia de género afecta más a las niñas y a las adolescentes, se sabe que también los niños y los adolescentes están en situación de riesgo. Las víctimas, cualesquiera que sean, pueden experimentar ansiedad, depresión, pérdida de autoestima, descenso del rendimiento, inadaptación escolar, etc. En casos extremos, se acumulan la sensación de soledad, el sufrimiento y la desesperanza, hasta el punto de que pueden conducir a pensar en el suicidio.

Cuando las conductas antisociales aparecen a una edad temprana y se vuelven más recurrentes y variadas (por ejemplo, peleas, hurtos, actos vandálicos) en la adolescencia, aunque no necesariamente, es más probable que favorezcan otros comportamientos de riesgo como el consumo de drogas y/o alcohol, que de no prevenirse pueden generar una dependencia que derive en alcoholismo o drogadicción. Estos comportamientos adictivos constituyen una amenaza para la salud pública (OMS, 2019) y su uso con frecuencia se vincula a conductas antisociales y a estilos de vida particulares como la organización en grupos para la perpetración de actos vandálicos e incluso, llevados al extremo, a conductas delictivas.

En el presente trabajo, más que el estudio de los jóvenes que ya tienen una trayectoria de delincuencia y de uso de sustancias psicoactivas (drogas y/o alcohol) que requiere medidas legales y de salud que generan aislamiento social para su tratamiento, nos centraremos en grupos de jóvenes que en su mayoría aún tienen un estilo de vida que les permite ir a la escuela y convivir con los docentes y compañeros, pero que sus prácticas de ocio involucran situaciones de riesgo vinculadas al consumo de drogas y/o alcohol. Desde luego resulta preocupante porque es sabido que dicho consumo, que suele realizarse en grupo, puede perjudicar el desarrollo madurativo y tener graves consecuencias en el rendimiento académico, en las relaciones sociales y en el conjunto de la vida de los adolescentes (Heradstveit, Skogen, Hetland y Hysing, 2017; Spear, 2018). Dado que, en general, los factores implicados en el consumo abusivo de drogas y/o alcohol suelen ser variados, algunos autores (Espada, Botvin, Griffin y Méndez, 2003; Biolatto, 2017), sugieren la conveniencia de intervenir a nivel familiar, escolar y social.

El objetivo general de este estudio consistió en analizar los principales problemas de convivencia desde la visión del alumnado de $3^{\circ}$ de Educación Secundaria Obligatoria (E.S.O.) en la ciudad de Ávila, España. Los objetivos específicos fueron: (a) Identificar el conocimiento del alumnado sobre experiencias de agresión, como acoso escolar o ciberacoso; (b) Identificar las preocupaciones de los alumnos sobre los problemas de convivencia relacionados con el vandalismo, la violencia de género y el racismo; (c) Conocer qué percepción tienen los alumnos sobre las situaciones de riesgo vinculadas al consumo de drogas y/o alcohol en el entorno escolar; (d) Proponer posibles líneas estratégicas de mejora de convivencia. 


\section{MÉTODO}

El estudio se llevó a cabo desde un enfoque cuantitativo, mediante un diseño exploratorio de corte transversal, en el que se aplicó un instrumento diseñado ad hoc a alumnos de $3^{\circ}$ de ESO en la ciudad de Ávila, España, sobre los principales problemas relacionados con problemas de convivencia.

\subsection{PARTICIPANTES}

La investigación se realizó con alumnos de $3^{\text {er }}$ Curso de Educación Secundaria Obligatoria (E.S.O.) de centros públicos de la ciudad de Ávila. La muestra quedó integrada por 116 alumnos, con edad media de 14,5 años, de los cuales 69 (59\%) son mujeres y 47 (41\%) son varones. En concreto se contó con la participación de los siguientes Institutos de Educación Secundaria (I.E.S.): (1) I.E.S. Isabel de Castilla ( $n=22)$; (2) I.E.S. Vasco de la Zarza $(n=44)$; (3) I.E.S. Alonso de Madrigal ( $n=23)$; (4) I.E.S. José Luis López Aranguren (n=27). El nivel socioeconómico de las áreas de influencia de los Centros es de clase media.

\subsection{INSTRUMENTO}

Se diseñó un instrumento de 24 ítems (Rodrigo, 2015) a fin de analizar la percepción de los jóvenes sobre los principales problemas de convivencia escolar, a partir de la Instrucción 7/2013 de la Secretaría de Estado de Seguridad sobre el Plan Director para la convivencia y mejora de la seguridad en los centros educativos y sus entornos. El instrumento fue sometido a la revisión por parte de expertos; docentes universitarios avalados por investigaciones relacionadas con el tema y probado en una muestra piloto. A continuación, se indican las Dimensiones que conformaron el estudio:

Experiencias de agresión: (1) Acoso escolar: conocimiento del término y de experiencias (3 ítems); (2) Ciberacoso: uso de redes sociales, visión de riesgo y experiencias (5 ítems).

Problemas de convivencia: (1) Vandalismo: bandas juveniles, hurtos y actos vandálicos (3 ítems); (2) Violencia de género: conocimiento del término y de experiencias (3 ítems); (3) Violencia racista: experiencias (2 ítems).

Conductas de riesgo: consumo de drogas y/o alcohol en el entorno escolar (5 ítems).

En función de las preguntas de cada dimensión, los ítems son valorados mediante una escala de respuesta tipo Likert, así como preguntas de opción múltiple.

\subsection{PROCEDIMIENTO}

La recolección de los datos se llevó a cabo de manera colectiva, después de contar con la autorización de las autoridades escolares sobre el procedimiento y objetivos del estudio. Se aseguró a los participantes la confidencialidad de los datos y su participación fue completamente voluntaria.

Se han efectuado cómputos de frecuencias y de porcentajes, así como una comparación estadística de proporciones respecto al género (Hombre / Mujer), utilizando el paquete estadístico SPSS 21. Los resultados se basan en pruebas bilaterales con un nivel de significación $(\mathrm{p}<0,05)$, utilizando para ello la corrección de Bonferroni en todas las comparaciones. 


\subsection{CONSIDERACIONES ÉTICAS}

La Investigación, como Trabajo fin de Máster de formación final de Inspectores de Policía, fue aprobado por la Coordinación de Formación de la Escuela Nacional de Policía (ÁvilaEspaña) en febrero de 2015.

\section{RESULTADOS}

\subsection{EXPERIENCIAS DE AGRESIÓN}

\subsubsection{Acoso escolar (definición y conocimiento de experiencias)}

En cuanto al término acoso escolar, la mayoría del alumnado $(98,3 \%)$ lo definen como "maltratar a un compañero bien sea verbal, física o psicológicamente de forma reiterada a lo largo de un tiempo determinado" y sólo unos cuantos $(1,7 \%)$ lo definen como "Pegar y/o insultar a algún compañero de vez en cuando si te cae mal". Respecto al conocimiento de experiencias de acoso escolar en el centro educativo, el 55,3\% de chicos y 62,3\% de chicas han tenido conocimiento de "algún" o "varios casos", con diferencias significativas $(\mathrm{p}<0,05)$ en la respuesta de conocimiento de "varios casos"; las chicas han tenido un mayor conocimiento de varios casos de acoso escolar $(13,0 \%)$ que los chicos $(2,1 \%)$.

\subsubsection{Ciberacoso (uso de redes sociales, visión de riesgo y experiencias)}

Respecto al uso de redes sociales, las chicas las utilizan en mayor medida $(98,6 \%)$ que los chicos $(87,2 \%)$, existiendo diferencias significativas entre ambos $(p<0,05)$. Las redes más utilizadas en general (y en las que no existen diferencias significativas por género) son: Twitter (26,6\%), Facebook (20,5\%) y Tuenti $(17,1 \%)$. Respecto a otras redes $(35,7 \%)$, de mayor a menor utilización son las siguientes: Instagram, WhatsApp, Snapchat y Ask.fm. Asimismo, existen diferencias significativas por género $(p<0,05)$; las chicas utilizan otras redes en mayor medida $(38,5 \%)$ que los chicos $(31,4 \%)$.

En lo que respecta a la percepción de riesgo, no existen diferencias significativas por género y en su mayoría $(88,2 \%)$ los jóvenes reconocen riesgos en la utilización de las redes sociales. Sin embargo, más de la mitad consideran que las utiliza con seguridad $(57,8 \%)$. Respecto a las experiencias que dicen conocer de agresiones en redes sociales, casi un $80 \%$ manifiestan tener conocimiento o haber presenciado agresiones, independiente del género. De ellas, como se observa en la Tabla 1, destacan el Ciber-acoso (37,9\%); el Sexting $(36,8 \%)$ o el Child grooming $(25,3 \%)$. Por su parte, la percepción de delito de estas conductas es generalizada $(90,5 \%)$. 
Tabla 1. Conocimiento de agresiones en redes sociales

\begin{tabular}{|l|c|c|c|c|c|c|}
\hline \multirow{2}{*}{$\begin{array}{l}\text { ¿Conoces algunas situaciones que se } \\
\text { producen en Internet y que atentan contra } \\
\text { tu seguridad? }\end{array}$} & \multicolumn{6}{|c|}{ RESPUESTAS } \\
\cline { 2 - 8 } Género & \multicolumn{2}{|c|}{ Ciber-acoso } & \multicolumn{2}{|c|}{ Child grooming } & \multicolumn{2}{|c|}{ Sexting } \\
\hline Hombres & f & $\%$ & f & $\%$ & f & $\%$ \\
\hline Mujeres & 22 & 32,8 & 19 & 28,4 & 26 & 38,8 \\
\hline Total & 47 & 40,8 & 27 & 23,5 & 41 & 35,7 \\
\hline
\end{tabular}

Nota: Los porcentajes en cada fila suman $100 \%$

Fuente. Elaboración propia.

\subsection{PROBLEMAS DE CONVIVENCIA}

\subsubsection{Vandalismo (bandas juveniles, hurtos y actos vandálicos)}

En cuanto a la presencia de bandas juveniles, el 75,9\%, e independientemente del género, manifiestan no haber recibido agresiones por parte de bandas juveniles, 22,4\% alguna vez y $1,7 \%$ en muchas ocasiones. Por su parte, el 74,1\% manifiestan que nunca han sufrido hurtos en su entorno escolar, $25,0 \%$ alguna vez y 9,0\% muchas veces. Lo más común es haber presenciado actos vandálicos en el entorno escolar, alguna vez $(62,1 \%)$ o muchas veces $(12,9 \%)$, observándose diferencias significativas por género en este último tipo de respuesta $(p<0,05)$; un mayor número de chicos han presenciado actos vandálicos muchas veces $(23,4 \%)$ en comparación con las chicas $(5,48 \%)$.

\subsubsection{Violencia de género (conocimiento del término y de experiencias)}

La violencia de género es considerada por la mayoría de los alumnos $(97,4 \%)$ como: "Un tipo de violencia física o psicológica ejercida contra las mujeres, que impacta de manera negativa en su identidad y bienestar social, físico y/o psicológico”. Por su parte, la percepción de la existencia de algunas situaciones de violencia de género entre compañeros es reconocida, independientemente del género, por el $62,1 \%$ de los encuestados, mientras que sólo un 6,9\% considera que sucedan frecuentemente y 31,0\% que no sucedan nunca.

En cuanto a las experiencias de maltrato hacia compañeras parece que son minoritarias, el 66,4\% opina que no ha presenciado este tipo de actos, 31,0\% sólo en algunas ocasiones y 2,6\% frecuentes. Como se observa en la Tabla 2, entre estas experiencias las más comunes son "insultos, gritos y voces", encontrándose diferencias significativas respecto a los otros tipos $(p<0,05)$. 
Tabla 2. Violencia de género entre compañeros

\begin{tabular}{|c|c|c|c|c|c|c|}
\hline \multirow{2}{*}{$\begin{array}{l}\text { ¿Has presenciado alguna vez alguna } \\
\text { situación de violencia o maltrato hacia } \\
\text { una chica por parte de su pareja o de } \\
\text { algún compañero? }\end{array}$} & \multicolumn{6}{|c|}{ RESPUESTAS } \\
\hline & \multicolumn{2}{|c|}{$\begin{array}{l}\text { Insultos, gritos, } \\
\text { voces* }\end{array}$} & \multicolumn{2}{|c|}{$\begin{array}{l}\text { Empujones o } \\
\text { golpes }\end{array}$} & \multicolumn{2}{|c|}{$\begin{array}{c}\text { Tocamiento } \\
\text { sexual }\end{array}$} \\
\hline Género & $f$ & $\%$ & $f$ & $\%$ & $\mathrm{f}$ & $\%$ \\
\hline Hombres & 6 & 35,3 & 4 & 23,5 & 7 & 41,2 \\
\hline Mujeres & 24 & 50,0 & 11 & 22,9 & 13 & 27,1 \\
\hline Total & 69 & 46,2 & 15 & 23,1 & 19 & 29,2 \\
\hline
\end{tabular}

*Diferencias significativas $(p<0,05)$; los porcentajes en cada fila suman $100 \%$

Fuente. Elaboración propia.

\subsubsection{Violencia Racista (experiencias)}

Respecto a las experiencias de violencia racista, poco más de la mitad de los alumnos $(51,7 \%)$, con independencia del género, manifiesta que en algunas ocasiones ha presenciado actos de violencia racista, $6,9 \%$ señala que frecuentemente, aunque $41,4 \%$ nunca ha presenciado este tipo de actos. Como se observa en la Tabla 3, entre las experiencias presenciadas violencia racista destacan las verbales $(50,5 \%)$ y en un segundo lugar las discriminatorias $(35,6 \%)$.

Tabla 3. Violencia racista entre compañeros

\begin{tabular}{|c|c|c|c|c|c|c|}
\hline \multirow{3}{*}{$\begin{array}{l}\text { ¿Has presenciado alguna vez en el } \\
\text { entorno de tu centro algún tipo de } \\
\text { agresión hacia algún compañero por el } \\
\text { hecho de ser de otra raza u otro país? } \\
\text { Género }\end{array}$} & \multicolumn{6}{|c|}{ RESPUESTAS } \\
\hline & \multicolumn{2}{|c|}{ Insultos } & \multicolumn{2}{|c|}{$\begin{array}{c}\text { Humillarle, aislarle, } \\
\text { discriminarle }\end{array}$} & \multicolumn{2}{|c|}{$\begin{array}{l}\text { Agresiones } \\
\text { físicas }\end{array}$} \\
\hline & $\mathrm{f}$ & $\%$ & $\mathrm{f}$ & $\%$ & $\mathrm{f}$ & $\%$ \\
\hline Hombres & 19 & 51,4 & 12 & 32,4 & 6 & 16,2 \\
\hline Mujeres & 32 & 50,0 & 24 & 37,5 & 8 & 12,5 \\
\hline Total & 51 & 50,5 & 36 & 35,6 & 14 & 13,9 \\
\hline
\end{tabular}

Nota: Los porcentajes en cada fila suman $100 \%$

Fuente. Elaboración propia. 


\subsection{CONDUCTAS DE RIESGO}

\subsubsection{Consumo de drogas y alcohol en el entorno escolar}

En lo referente al consumo de drogas, como se muestra en la Tabla 3, la mayoría del alumnado $(76,7 \%)$, independientemente del género, dice haber presenciado alguna o muchas veces consumir drogas cerca del centro escolar. Sin embargo, mayoritariamente $(69,8 \%)$ manifiestan que no han presenciado la venta de drogas en el entorno escolar, aunque 3,2\% sí lo ha hecho. Asimismo, frente a la pregunta de si es fácil adquirir droga en el entorno del centro, el 55,2\% opina que no, pero el $44,8 \%$ dice que sí.

Tabla 4. Percepción de consumo de drogas en el entorno del centro escolar

\begin{tabular}{|l|c|c|c|c|c|c|}
\hline \multirow{2}{*}{$\begin{array}{l}\text { ¿Has visto alguna vez que en el entorno } \\
\text { de tu centro escolar se consuma algún } \\
\text { tipo de droga? }\end{array}$} & \multicolumn{2}{|c|}{ RESPUESTAS } \\
\cline { 2 - 8 } & \multicolumn{2}{|c|}{ Nunca } & \multicolumn{2}{c|}{ Alguna vez } & \multicolumn{2}{c|}{ Muchas veces } \\
\hline Género & $\mathrm{f}$ & $\%$ & $\mathrm{f}$ & $\%$ & $\mathrm{f}$ & $\%$ \\
\hline Hombres & 10 & 21,3 & 23 & 48,9 & 14 & 29,8 \\
\hline Mujeres & 21 & 30,4 & 35 & 50,7 & 13 & 18,8 \\
\hline Total & 31 & 26,7 & 58 & 50,0 & 27 & 23,3 \\
\hline
\end{tabular}

Nota: Los porcentajes en cada fila suman $100 \%$

Fuente. Elaboración propia.

Sobre el consumo de alcohol entre compañeros, como se muestra en la Tabla 4, la mayoría (75,8\%, e independiente del género) consideran que éste está presente entre ellos de forma esporádica y, en un menor porcentaje $(24,1 \%)$ muy frecuentemente. Por otro lado, la mayoría $(66,4 \%)$ reconoce que el consumo de alcohol es perjudicial en cualquier modalidad de consumo para su edad. No obstante, el 32,8\% vincula el daño del alcohol sólo al consumo diario.

Tabla 5. Percepción de consumo de alcohol entre compañeros

\begin{tabular}{|l|c|c|c|c|c|c|}
\hline \multirow{2}{*}{ ¿Los compañeros de tu edad beben alcohol? } & \multicolumn{6}{|c|}{ RESPUESTAS } \\
\cline { 2 - 8 } & \multicolumn{2}{|c|}{ Nunca } & \multicolumn{2}{|c|}{ Algunas veces } & \multicolumn{2}{c|}{ Muchas veces } \\
\hline Género & $\mathrm{f}$ & $\%$ & $\mathrm{f}$ & $\%$ & $\mathrm{f}$ & $\%$ \\
\hline Hombres & 6 & 12,8 & 29 & 61,7 & 12 & 25,5 \\
\hline Mujeres & 1 & 1,4 & 52 & 75,4 & 16 & 23,2 \\
\hline Total & 7 & 6,0 & 81 & 69,8 & 28 & 24,1 \\
\hline
\end{tabular}

Nota: Los porcentajes en cada fila suman $100 \%$

Fuente. Elaboración propia. 


\section{DISCUSIÓN Y CONCLUSIONES}

A continuación, se discuten los principales resultados, siguiendo las Dimensiones estudiadas.

\subsection{EXPERIENCIAS DE AGRESIÓN}

En lo referente al acoso escolar, los resultados nos llevan a inferir que éste es reconocido por la mayoría del alumnado investigado y está presente en el día a día de los escolares de estos Institutos, ya que más de la mitad de los encuestados, independientemente del género, manifiesta haber conocido algún caso de acoso escolar en su centro y un preocupante porcentaje $(8,6 \%)$ manifiesta que ha conocido varios casos. Respecto al ciberacoso, a partir de los hallazgos podemos decir que las redes sociales son muy utilizadas por el alumnado y reconocen la existencia de riesgos en su utilización. Las Redes, según orden de mayor a menor utilización serían: Twitter, Facebook, Tuenti, Instagram, WhatsApp, Snapchat y Ask.fm. Casi un $80 \%$ de los estudiantes conoce situaciones peligrosas en Internet y especialmente alarmante resulta que uno de cada cuatro conozca situaciones de child grooming o acoso sexual a menores a través de Internet; un grave problema que afecta a ambos géneros, aunque la investigación revela que la solicitación sexual y las interacciones sexuales con adultos tienden a ser más frecuentes entre chicas (De Santisteban y GámezGuadix, 2018).

Estos datos incitan a proceder más decididamente en los esfuerzos para prevenir el acoso y el ciberacoso, a través de orientaciones estratégicas para el actuar educativo en las que se plasme el compromiso auténtico con la convivencia, ya que el impacto del acoso puede ser devastador tanto en los escolares que lo sufren como en los que lo infligen y en los que lo observan. Al respecto, el Ministerio de Educación y Formación Profesional en colaboración con la Federación Española de Municipios y Provincias (FEMP, 2019) han buscado difundir distintos programas innovadores y eficaces desarrollados en distintos municipios españoles en la prevención e intervención del acoso escolar. Por su parte, Luengo (2014) ofrece claves a los centros escolares para impulsar la alfabetización digital de los menores que contribuya a la prevención del ciberacoso.

\subsection{PROBLEMAS DE CONVIVENCIA}

En cuanto a los aspectos relativos a las bandas juveniles, uno de cada cuatro encuestados manifiesta haber sido víctima o haber presenciado agresiones por parte de estos grupos. Por lo que podemos decir, con base en estos resultados, que sí existen estos grupos en la ciudad de Ávila. Por lo tanto, desde los servicios policiales se deberían incrementar la vigilancia y el control de estos grupos, conociendo quiénes son sus miembros y qué actividades realizan. Asimismo, como bien señala Krauskopf (2006), los centros escolares deben establecer oportunas medidas preventivas y remediales, por ejemplo, a través de protocolos apropiados que eviten la aparición de problemas y que posibiliten la búsqueda ágil de soluciones eficaces en cuanto se detectan.

En esta misma línea se encuentra lo relativo a los robos o hurtos en el entorno escolar; tres de cada cuatro alumnos nunca han sufrido esta actividad delictiva, sin embargo, un alumno de cada cuatro manifiesta que alguna vez ha experimentado un robo 
o hurto en el centro escolar. Es por ello necesario que desde la dirección de los centros se establezca un plan de actuación educativa, además de estrechar la colaboración con los servicios policiales para identificar qué personas se dedican a realizar estas actividades delictivas en el entorno escolar. Además, en lo relativo a los actos vandálicos realizados en el entorno escolar, tres de cada cuatro encuestados manifiestan haberlos presenciado en alguna ocasión, dato que resulta sorprendente y preocupante. Por ello, uno de los objetivos prioritarios de intervención preventiva y remedial de los centros educativos debe centrarse en este aspecto (Torkaman y Saei, 2016).

Respecto a la violencia de género, a partir de los resultados, es preocupante que una mayoría de alumnos consideren que este tipo de situaciones sí se dan entre los jóvenes de su edad y de extrema gravedad resulta que el 33,6\% de los encuestados haya presenciado directamente violencia o maltrato hacia alguna mujer por parte de su pareja o por parte de algún compañero del centro escolar. Este dato resulta alarmante ya que incluso a edades tan tempranas como la adolescencia ( $3^{\circ}$ E.S.O.) las situaciones de violencia de género están presentes con cierta frecuencia. Resulta así de especial trascendencia la intervención de los poderes públicos con campañas informativas y formativas para prevenir estas situaciones e inculcar a los menores la igualdad entre hombres y mujeres.

Asimismo, resulta conveniente informar a las menores sobre la necesidad de interponer denuncia si son víctimas de estas situaciones. Es fundamental que tanto profesores como el conjunto de personal administrativo de los centros escolares estén muy atentos a este tipo de situaciones y las pongan inmediatamente en conocimiento de los servicios policiales para que se pueda intervenir si se está produciendo una situación de violencia de género. Como es sabido, el papel del profesorado es clave en la convivencia escolar (García-Vidal, Sola y Peiró, 2016), así como la participación activa de las familias (Martínez-Otero, 2018; Sánchez, Daura y Laudadío, 2019). Se precisa por tanto una coordinada y eficaz intervención por parte de toda la comunidad educativa: padres y madres, profesores, los propios alumnos, con la oportuna coordinación de los poderes públicos, para prevenir y canalizar estas conductas tan lesivas y extendidas en los centros escolares.

En cuanto a la violencia racista, más de la mitad de los alumnos han sido testigos de agresiones de tipo racista en el entorno de su centro escolar. Según expresan los alumnos estas agresiones fueron insultos, humillaciones y actos de discriminación y en menor grado los encuestados han sido testigos de agresiones físicas de tipo racista entre compañeros de Instituto. Estos resultados nos hacen ver la necesidad de fomentar la igualdad entre personas de distintas razas y culturas (García-Medina, 2018), siendo necesario que desde los centros educativos se trabaje por la integración de los alumnos extranjeros con campañas de educación intercultural en las que se explique a los jóvenes la gravedad de las conductas de tipo racista y la urgencia de erradicarlas de los centros educativos y de la sociedad en general, así como la relevancia de convivir todos en armonía independientemente de la raza, cultura o religión de cada persona.

También es labor de los educadores, que han de recibir oportuna formación intercultural e inclusiva, contribuir a la prevención del racismo, la xenofobia y cualquier otro tipo de discriminación. Como señala Lepe-Carrión (2018), muchos de los problemas que asociamos al racismo están imbricados en una red más compleja de relaciones, donde la escuela, los programas y sus respectivas prácticas pedagógicas en contexto intercultural se constituyen en piezas fundamentales. 


\subsection{CONDUCTAS DE RIESGO}

En primer lugar, conviene llamar la atención sobre el hecho de que ciertas conductas de riesgo, como el consumo de drogas y/o alcohol, facilitan en la etapa de la adolescencia la realización de otras acciones igualmente de riesgo, por ejemplo, de índole sexual (Alfonso y Figueroa, 2017). En cuanto a la venta de drogas, la mayoría de los encuestados $(70,0 \%)$ dice no haber presenciado que se venda droga en el entorno escolar, aunque para el 30,0\% esas situaciones de venta existen y además casi la mitad de los encuestados manifiestan que es fácil comprar drogas en su centro escolar.

Por otra parte, los datos del estudio sugieren que el consumo de drogas es algo habitual en los entornos de los centros escolares analizados, así lo manifiestan tres de cada cuatro alumnos; dato que resulta cuando menos sorprendente y a la vez preocupante, por lo que las campañas y charlas formativas deberían incidir en los perjuicios del consumo de estas sustancias. Coincidimos con otros investigadores (Krauskopf, 2006; Penalva, 2018; Spear, 2018) en la necesidad de fomentar oportunidades para que los adolescentes, como sujetos sociales, desarrollen sus capacidades y asimismo se impulsen los medios seguros para la prevención de estas conductas de riesgo con estrategias desde la familia, la escuela y la comunidad que permitan orientar y acompañar a los jóvenes.

Como dato esperanzador podemos resaltar que el 70,0\% de los encuestados nunca ha presenciado que se vendan drogas en las inmediaciones de su centro escolar, aunque para el 30,0\% esas situaciones de venta existen por lo que éste es un dato muy relevante. De igual modo casi la mitad de los encuestados manifiestan que es fácil comprar drogas en su centro escolar, por lo que podemos deducir que saben dónde o a quién las podrían comprar; esto nos hace reafirmar la existencia de puntos minoristas de venta de estupefacientes o la existencia de personas que se dedican a la venta en el entorno escolar.

En cuanto al consumo de alcohol también resulta preocupante que el 94,0\% de los encuestados digan que los compañeros de su edad (alumnos de $3^{\circ}$ de E.S.O.) beben alcohol. Por lo que resulta de vital importancia que las campañas educativas incidan sobre los perjuicios de este consumo, aunque, como aspecto positivo podemos concluir que la práctica totalidad de los encuestados consideran como perjudicial el consumo de alcohol. Sin embargo, un 32,8\% de los alumnos sólo lo consideran perjudicial si se consume diariamente. Esto nos lleva a inferir que los menores consumen alcohol aun a sabiendas de los daños que causa a la salud, por lo cual se deben redoblar los esfuerzos preventivos al respecto, como bien indican Rodríguez, Sanchiz y Bisquerra (2014).

Por último, un dato destacable a nivel global en cuanto a las diferencias de género es que, en general, no se han encontrado diferencias significativas entre los resultados referidos a los chicos y las chicas, salvo en tres campos: en el de experiencias de conocimiento de acoso escolar, en la utilización de Redes sociales y en haber presenciado actos vandálicos; en los dos primeros casos son mayores entre el alumnado femenino y en el último entre el masculino. Estos resultados nos orientan a profundizar más en trabajos futuros sobre estos problemas de convivencia escolar desde una perspectiva de género.

En relación con las limitaciones del presente estudio, es importante señalar que al tratarse de un estudio exploratorio no es posible la generalización de los hallazgos. Sin embargo, los datos obtenidos en los cuatro institutos, desde la mirada de los propios actores, son orientadores respecto a los problemas de convivencia escolar en la ESO de la ciudad de Ávila. A la luz de los resultados, la investigación se podría ampliar a un número mayor 
de alumnos o comparar con otros centros de distinto nivel socio-económico o de otras localidades, etc. Así también, podríamos considerar aspectos que completen el presente estudio, como realizar entrevistas a los estudiantes, que nos permita profundizar en el conocimiento y comprensión de este objeto de estudio. Además de introducir como fuente de información (mediante cuestionarios o entrevistas preparados para tal fin) la visión de los padres o tutores en su función como educadores, que posibilite una mayor comprensión del fenómeno y el posterior diseño de proyectos preventivos, contextualizados según las necesidades de cada comunidad educativa.

\section{SÍNTESIS Y CONCLUSIONES}

Con base en los hallazgos del presente estudio, consideramos que desde una perspectiva socio-contextual amplia entre el alumnado de $3^{\circ}$ de ESO estudiado, los problemas de convivencia están poco arraigados, aunque existirían cuatro campos básicos de mejora y sobre los que se debería incidir en las campañas formativas dirigidas a los menores comprendidos en estas edades estudiadas:

La prevención y detección del acoso escolar que aunque residual aparece latente entre el alumnado estudiado.

La profundización sobre los riesgos que entraña el uso indiscriminado de las redes sociales con especial incidencia de perspectiva de género (dado que son más utilizadas por las chicas).

La educación sobre la importancia de la igualdad, tanto entre hombres y mujeres para evitar comportamientos de violencia de género, como entre todas las personas con independencia de su raza o país de nacimiento con el objetivo de evitar los actos racistas o xenófobos que en ocasiones sí aparecen presentes en los grupos estudiados.

Profundizar sobre el conocimiento de los perjuicios del consumo de drogas y alcohol que aunque no se ha manifestado como un problema actual sí es un campo de posible incremento de consumos no deseados para el colectivo estudiado.

Así pues, ante la complejidad de que las intervenciones logren ser efectivas, desde una visión sistémica para la promoción de mejores formas de convivencia, coincidimos con otros investigadores (Cardozo-Rusinque, Martínez-González, De la Peña-Leiva, Avedaño-Villa y Crissien-Borrero, 2019) en que los escenarios que conforman los niveles micro-sociales (familia-escuela) y meso-sociales (barrio-comunidad), son esenciales en la solución pacífica de los conflictos, para establecer redes de apoyo, afianzar la formación moral, y ser modelos legítimos de convivencia que favorezcan una sana adaptación social de niños y jóvenes. De ahí que se requerirá de la suma de las distintas voluntades para construir nuevos significados y experimentar formas de vivir juntos en las que cada persona tenga un lugar.

En suma, la escuela ha de abrir las puertas a la ayuda, la solidaridad, la comprensión, etc.; acciones todas que podemos calificar como prosociales y que constituyen la mejor vacuna contra sus opuestas: las conductas antisociales. Complementariamente, la consolidación de la orientación en los centros escolares contribuye también a la prevención de estos problemas, así como de las conductas de riesgo. La prevención y el fomento de estrategias apropiadas requiere en sintonía con lo planteado por Klimenko, Plaza, Bello, García y Sánchez (2018), un acercamiento a las familias y a la comunidad, partiendo de 
premisas de una educación para la vida -que posibilite al alumnado aprender a aprender, aprender a hacer, aprender a ser y aprender a convivir- y de la incorporación de actitudes proactivas que impulsen la convivencia desde una acción educativa integral.

\section{REFERENCIAS BIBLIOGRÁFICAS}

Alfonso, L. y Figueroa, L. (2017). Conductas sexuales de riesgo en adolescentes desde el contexto cubano. Revista Ciencias Médicas de Pinar del Río, 21(2), 193-301. Recuperado de <http:// scielo.sld.cu/pdf/rpr/v21n2/rpr20217.pdf>

Arenas, L. (2013). Sexismo en adolescentes y su implicación en la violencia de género. Boletín criminológico, (144), 1-5. Recuperado de <http://www.boletincriminologico.uma.es/ boletines/144.pdf>

Biolatto, L. (2017). Estrategias con adolescentes para prevenir el consumo problemático de alcohol: modalidades y ámbitos de aplicación. Hacia la Promoción de la Salud, 23(2), 48-66.

Bravo, A. I. y Herrera Torres, L. (2011). Convivencia escolar en Educación Primaria. Las habilidades sociales del alumnado como variable moduladora. Dedica. Revista de educação e humanidades, (1), 173-212. Recuperado de <http://revistaseug.ugr.es/index.php/dedica/article/ view/7166/6254>

Caetano, A. P., Amado, J., Martins, M. J., Veiga, A. M., Freire, I. y Ribeiro, M. T. (2017). Cyberbullying: motivos da agressão na perspetiva de jovens portugueses. Educação e Sociedade, 38(141), 1017 1034. Recuperado de <http://dx.doi.org/10.1590/ES0101-73302017139852>

Cardozo-Rusinque, A., Martínez-González, M. B., De la Peña-Leiva, A., Avedaño-Villa, I. \& Crissien-Borrero, T. (2019). Factores psicosociales asociados al conflicto entre menores en el contexto escolar. Educação e Sociedade, 40, 1-20. Recuperado de <http://dx.doi.org/10.1590/ es0101-73302019189140>

Castillo-Pulido, L. E. (2011). El acoso escolar. De las causas, origen y manifestaciones a la pregunta por el sentido que le otorgan los actores. Magis. Revista Internacional de Investigación en Educación, 4(8), 415-428. Recuperado de <http://www.redalyc.org/pdf/2810/281021722009. pdf $>$

Cerezo-Ramírez, F. (2012). Bullying a través de las TIC. Boletín Científico Sapiens Research, 2(2), 24-29. Recuperado de <https://dialnet.unirioja.es/servlet/articulo?codigo=3973451>

De Santisteban, P. y Gámez-Guadix, M. (2018). Prevalence and risk factors among minors for online sexual solicitations and interactions with adults. The Journal of Sex Research, 55(7), 939-950.

Espada, J. P., Botvin, G. J., Griffin, K. W. y Méndez, X. (2003). Adolescencia: consumo de alcohol y otras drogas [Adolescence: consumption of alcohol and other drugs]. Papeles del Psicólogo, 23(84), 8-17. Recuperado de <http://www.redalyc.org/pdf/778/77808402.pdf>

FEMP (2019). IV Catálogo de buenas prácticas municipales en la prevención frente al absentismo y el acoso escolar. Madrid: Federación Española de Municipios y Provincias; Ministerio de Educación y Formación Profesional. Recuperado de <https://sede.educacion.gob.es/publiventa/ descarga.action?f_codigo_agc=19832>

Garaigordobil, M. (2011). Prevalencia y consecuencias del cyberbullying: una revisión. International Journal of Psychology and Psychological Therapy, 11(2), 233-254. Recuperado de <http:// www.ijpsy.com/volumen11/num2/295/prevalencia-y-consecuenciasdel-cyberbullying-ES.pdf>

García-Medina, R. (2018). Inclusión y convivencia para prevenir el racismo, la xenofobia y otras formas de intolerancia: uno de los retos principales de nuestro sistema educativo. Ciencia, Técnica y Mainstreaming Social, (2), 53-65.

García-Vidal, M., Sola, J. M. y Peiró, S. (2016). Los docentes como clave en la construcción de la convivencia escolar. Edetania, (50), 69-83. Recuperado de <https://revistas.ucv.es/index.php/ Edetania/article/view/19/19> 
Gázquez, J. J., Barragán, A. B., Pérez-Fuentes, M. C., Molero, M. M., Garzón, A. y Martos, Á. (2015). Factors associated with school violence: a systematic review. British Journal of Education, Society \& Behavioural Science, 11(3), 1-12.

Heradstveit, O., Skogen, J. C., Hetland, J. \& Hysing, M. (2017). Alcohol and Illicit Drug Use Are Important Factors for School-Related Problems among Adolescents. Frontiers in Psychology. doi: 10.3389/fpsyg.2017.01023

Ibarra, E. (2018). La mundialización de la intolerancia normaliza el odio identitario. En Movimiento contra la intolerancia. Informe Raxen Especial 2018. Madrid: Movimiento contra la intolerancia. Recuperado de <http://www.mitramiss.gob.es/oberaxe/ficheros/documentos/ informeraxenespecial2018.pdf $>$

Instrucción de la Secretaría de Estado de Seguridad. (7/2013). "Plan Director para la Convivencia y Mejora de la Seguridad en los Centros Educativos y sus Entornos". Recuperado de <http://www. interior.gob.es/documents/642012/1568685/Instruccion_7_2013.pdf/cef1a61c-8fe4-458d-ae0dca1f3d336ace>

Klimenko, O., Plaza, D., Bello, C., García, J. y Sánchez, N. (2018). Estrategias preventivas en relación a las conductas adictivas en adolescentes. Revista Psicoespacios, 12(20), 144-172. Recuperado de $<$ http://revistas.iue.edu.co/revistasiue/index.php/Psicoespacios/article/view/1043>

Krauskopf, D. (2006). Estado del arte de los programas de prevención de la violencia en ámbitos escolares. Washington: Organización Panamericana de la Salud-Organización Mundial de la Salud. Recuperado de <http://iris.paho.org/xmlui/handle/123456789/2732>

Lepe-Carrión, P. (2018). Educación, racismo cultural y seguridad nacional: la escuela intercultural en contextos de violencia. Educação e Pesquisa, 44, 1-19. Recuperado de http://dx.doi.org/10.1590/ S1678-4634201844174819

Luengo, J. A. (2014). Ciberbullying. Prevenir y actuar. Guía de recursos didácticos para centros educativos. Madrid: Colegio Oficial de Psicólogos de Madrid, Fundación ATRESMEDIA. Recuperado de <http://www.copmadrid.org/webcopm/recursos/CiberbullyingB.pdf>

Marías, J. (1996). Persona. Madrid: Alianza Editorial.

Martínez-Otero, V. (2018). Condicionantes de la violencia en la escuela. En Martín, J. y MartínezOtero, V. (Eds.). Violencia y educación (18-21). Madrid: Universidad Nebrija.

Molina, J. A. y Vecina, P. (2015). Bullying, ciberbullying y sexting ¿Cómo actuar ante una situación de acoso? Madrid: Pirámide.

Nicolás, J. J. (2011). Acoso escolar. Archivos de criminología, seguridad privada y criminalística, (7), 1-8. Recuperado de <https://dialnet.unirioja.es/ejemplar/298972>

OMS (2019). Organización Mundial de la Salud. Recuperado de https://www.who.int/es

Penalva, A. (2018). La convivencia escolar. Un reto del siglo XXI. Revista Electrónica de Investigación y Docencia (REID), (20), 41-58. Recuperado de <https://revistaselectronicas.ujaen.es/index. $\mathrm{php} / \mathrm{reid} /$ article/view/3450/3249>

Rodrigo, M. (2015). Un estudio relacionado con el plan director para la convivencia y mejora de la seguridad en los centros educativos y sus entornos en la ciudad de Ávila (TFM final de Inspector, inédito), Ávila, Escuela Nacional de Policía.

Rodríguez, F. D., Sanchiz, M. L. y Bisquerra, R. (2014). Consumo de alcohol en la adolescencia: Consideraciones médicas y orientaciones educativas. Salud mental, 37(3), 255-260.

Ruiz-Ramírez, R. y Ayala-Carillo, M.R. (2016). Violencia de género en instituciones de educación. Ra Ximhai, 12(1), 21-32. Recuperado de <https://dialnet.unirioja.es/servlet/ articulo?codigo $=6463993>$

Sánchez, C., Daura, F. T. y Laudadío, J. (2019). La comunidad escolar como medio para el desarrollo socio emocional de los alumnos. Un estudio de caso en la República Argentina. Estudios Pedagógicos, 45(3), 31-49.

Spear, L. P. (2018). Effects of adolescent alcohol consumption on the brain and behavior. Nature Reviews Neuroscience, 19, 197-214. 
Torkaman, M. y Saei, I. (2016). Factors affecting the incidence of vandalism among high school students in Tehran, district 5 Hezekiah. International Journal of Humanities Cultural Studies, Número especial febrero, 1358-1369.

Torner, M. (2016). Investigación sobre la incidencia del vandalismo en los centros de educación secundaria. Tesis Doctoral. Alcalá de Henares, Universidad de Alcalá.

UNESCO-IICBA (2017). School safety manual. Tools for teachers, Addis Ababa, UNESCO- IICBA.

Vilalta, C. J. y Fondevila, G. (2018). School vandalism in Mexico. Journal of School Violence, 18, 392-404. 
